\title{
Desafios da atenção primária à saúde durante a pandemia da COVID-19 no Brasil
}

\section{Challenges of primary health care during the COVID-19 pandemic in Brazil}

DOI: $10.46919 / \operatorname{archv2n3-009}$

Recebimento dos originais: 01/05/2021

Aceitação para publicação: 30/06/2021

Elisa Mileni de Sá Gomes

Discente de medicina. Centro Universitário de Belo Horizonte, UniBH, MG, Brasil.

E-mail: elisamileni@gmail.com

Larissa Veloso Hilarino

Discente de medicina. Centro Universitário de Belo Horizonte, UniBH, MG, Brasil.

E-mail: larissavhilarino@gmail.com

Taynah Regianni Furtado

Discente de medicina. Centro Universitário de Belo Horizonte, UniBH, MG, Brasil.

E-mail: taynahfurtado@gmail.com

\section{André Luiz Silva Alvim}

Enfermeiro. doutorando e mestre em Enfermagem pela Universidade Federal de Minas Gerais.

Professordo Centro Universitário Una. Contagem, MG, Brasil.

E-mail: andrealvim1@hotmail.com

\section{RESUMO}

Objetivo: Levantar na literatura os desafios enfrentados pela APS em relação ao atendimento das demandas específicas de pacientes acometidos pela COVID-19. Métodos: Trata-se de uma revisão integrativa da literatura realizada nas bases de dados LILACS, MEDLINE, PubMed e na biblioteca SciELO. Resultados: Foi obtida uma amostra de sete artigos de acordo com os critérios de inclusão pré-estabelecidos. Considerando a análise dos artigos finais, observou-se que a APS tem seu potencial limitado diante da pandemia vigente principalmente quando se analisa a escassez de investimento financeiro nesse setor. Discussão: A Atenção Primária à Saúde apresenta falhas quanto a consolidação do teleatendimento, a distribuição de recursos, a organização e o planejamento dos centros de saúde e a execução da vigilância epidemiológica. Todos esses fatores comprometem a prevenção e o tratamento da COVID-19. Conclusão: As dificuldades enfrentadas pela APS na pandemia da COVID-19 são reflexo da má gestão da saúde pública em um cenário anterior à tal crise sanitária.

Palavras-chave: Atenção Primária à Saúde, Infecções por Coronavirus; COVID-19, Sistema Único de Saúde; Brasil.

\begin{abstract}
Objective: To raise in the literature the challenges faced by PHC in relation to attend the specific demands of patients affected by COVID-19. Methods: This is an integrative review of the literature based on the LILACS, MEDLINE, Pubmed and Scielo databases. Results: A sample of seven articles was obtained according to the pre-established inclusion criteria. Considering the analysis of the final articles, it was observed that PHC has its potential limited in face of the current pandemic, especially when analyzing the scarcity of financial investment in this sector. Discussion: Primary Health Care presents flaws regarding the consolidation of the healthcare phone system, the distribution of resources, the organization and
\end{abstract}


planning of health centers and the implementation of epidemiological surveillance.All these factors compromise the prevention and treatment of COVID-19. Conclusion: The difficulties faced by PHC in the COVID-19 pandemic are a reflection of the poor management of public health in a scenario prior to the health crisis.

Keywords: Primary Health Care, Coronavirus Infections, COVID-19, Unified Health System, Brazil.

\section{INTRODUÇÃO}

O sistema de atendimento à saúde da população se baseia na otimização do cuidado na comunidade para diminuir as disparidades entre os subgrupos, maximizando o acesso a esse direito pelos brasileiros (STARFIELD, 2004). Em termos históricos, a Conferência Internacional de Atenção Primária à Saúde de Alma-Ata, em 1978, discutiu justamente pautas em relação ao acesso integral à saúde, enfatizando-o como direito fundamental a ser garantido pelo governo. Como definido em Alma-Ata, a Atenção Primária à Saúde (APS) deve representar o primeiro contato do paciente ao buscar um atendimento universal e integral aos indivíduos, incluindo, além do setor da saúde, todas as áreas relacionadas ao desenvolvimento comunitário (BRASIL, 1978), estabelecendo como características essenciais a associação da saúde ao desenvolvimento econômico-social, a APS como primeiro contato do indivíduo ao sistema de atendimento e o acesso universal e participação social (GIOVANELLA, 2018).

No entanto, no Brasil, a APS não foi implementada seguindo tais termos à risca, visto que, apesar de serem condizentes aos fundamentos defendidos pelo atual Sistema Único de Saúde (SUS), estes se tornaram financeiramente inviáveis a princípio (GIOVANELLA, 2018). Com isso, uma nova designação foi implementada, a chamada Atenção Básica, buscando se afastar de uma abordagem seletiva que vinha sendo difundida por algumas entidades internacionais, pelas quais essa área do sistema era vista como uma etapa da atenção voltada, majoritariamente, para a população de baixa renda, oferecendo cuidados para casos de baixa complexidade (GIOVANELLA, 2018; OLIVEIRA; PEREIRA, 2013).

Em resumo, a atenção primária no país deu origem ao SUS. Entretanto, os serviços de saúde brasileiros ainda enfrentam dificuldades quanto à fragmentação e interrupção assistencial (OLIVEIRA; PEREIRA, 2013). Assim, esse serviço de saúde atua no acolhimento no âmbito individual e coletivo, a partir do conhecimento de todos os seus determinantes, sejam eles meios sociais ou físicos, além do cuidado com as enfermidades desse grupo, buscando compreender as demandas recebidas e o contexto em que elas se inserem (STARFIELD, 2004). Por meio dos preceitos do SUS e seus fundamentos, as unidades da atenção primária atuam na base do atendimento universal e contínuo à saúde, oferecendo medidas de prevenção de doenças, diagnóstico, reabilitação e manejo de problemas mais comuns na comunidade, estabelecendo alternativas para o bem-estar e melhora da qualidade de vida. Dessa maneira, é definida por meio de uma logística democrática e participativa que indica o território a ser abrangido por cada unidade, 
sendo esta a responsável sanitária pela resolução de problemas mais frequentes e de considerável impacto ocorridos na área (BRASIL, 2013a).

Em relação à pandemia do novo Coronavírus, independentemente do nível do serviço de saúde procurado pelo paciente, deve-se haver a comunicação com as unidades de atenção primária como forma de monitorar a evolução dos infectados e avaliar o tipo de tratamento prescrito, além de favorecer um controle estatístico dos casos locais. Tal medida visa avaliar as condições em que a comunidade está submetida e orientar o paciente sobre o período de afastamento, de maneira a diminuir a transmissibilidade da doença. Portanto, seja por demanda espontânea, entrada por busca ativa de paciente sintomático ou encaminhamento de usuário pela telemedicina, o atendimento pelo uso da APS deve ser priorizado em momentos preliminares (BRASIL, 2020b).

O vírus denominado SARS-CoV-2, primeiramente descrito na China em dezembro de 2019, provocou a pandemia do coronavírus ao longo do ano de 2020, atingindo todos os continentes do mundo e gerando milhares de vítimas. Segundo dados da Universidade Johns Hopkins, até o dia 19 de agosto de 2020, mais de 3.407.354 brasileiros se infectaram com tal doença, fazendo do Brasil o segundo país com o maior número de casos (JOHNS HOPKINS UNIVERSITY, 2020). Segundo informações do Ministério da Saúde, a taxa de letalidade dessa infecção atingiu 3,2\% também como base o dia 19 de agosto de 2020 (BRASIL, 2020a). A expansão do vírus e sua alta taxa de transmissão fizeram com que os países buscassem normas de isolamento social forçado, impondo medidas de prevenção de contágio.

Além do desconhecimento, a princípio, dessa nova doença, a comunidade médica e científica tem alertado sobre as possibilidades de evolução dessa doença que pode ser letal. Os sintomas da COVID-19 são caracterizados pela febre, tosse e fadiga, acompanhados ou não de sintomas menos comuns como diarreia, dor de cabeça, dispneia, entre outros (ROTHAN et al., 2020). O risco de desenvolvimento de Síndrome Respiratória Aguda Grave ao longo da infecção, principalmente àqueles indivíduos que compõem o grupo de risco, evidencia a probabilidade de evolução grave aos pacientes acometidos por esse vírus e a importância do monitoramento e atenção aos sinais de alerta de piora dos sintomas.

Frente ao risco de comprometimento pulmonar e cardiovascular que pode ser provocado pelo coronavírus, evidencia-se a importância do sistema de saúde junto ao enfrentamento da pandemia. Os profissionais de saúde compõem papel de destaque no combate direto ao vírus e também na orientação quanto às medidas de controle e prevenção da doença. Em soma, às atribuições anteriormente citadas, é possível incluir a função dos Centros de Saúde nesse embate, através da criação de planos de gerenciamento de risco com o objetivo de reforçar ações locais de manejo epidemiológico (MEDINA et al., 2020).

Dessa forma, é possível estabelecer uma ordenação à atuação da Atenção Primária nessa perspectiva de pandemia, seguindo as vertentes "vigilância em saúde nos territórios; atenção aos usuários com COVID19; suporte social a grupos vulneráveis e continuidade das ações próprias da APS”. Dentro desse contexto, 
enfatiza-se o cuidado necessário aos pacientes acometidos pelo vírus e suas particularidades, o que impõe atenção específica a seu estado de saúde, visto que dados da Organização Mundial da Saúde (OMS) comprovam que cerca de $80 \%$ dos indivíduos que contraíram tal doença manifestam formas assintomáticas ou sintomatologia leve, enquanto $15 \%$ dos pacientes requerem uso de oxigenoterapia e $5 \%$ exigem atendimento em uma Unidade de Terapia Intensiva (UTI) (WORLD HEALTH ORGANIZATION, 2020).

É de entendimento comum o fato de a APS não ser capaz de atuar diretamente com casos de grande complexidade da doença ou lidar de forma eficaz com a sua letalidade. Entretanto, a atenção primária, sendo sólida em relação à organização interna e à profissionais qualificados, tem um impacto relevante na população adscrita no que tange a atuação no cuidado e acompanhamento desse grupo. Ademais, o serviço primário da rede de saúde também se destaca no controle epidemiológico em termos estatísticos, sendo possível estabelecer protocolos de atuação frente à pandemia a partir dos dados locais (DAUMAS et al., 2020; PORTELA et al., 2020). Tendo em vista sua importância, a atuação plena da APS só pode ser assegurada nesse contexto com recursos estruturais e insumos adequados, visando garantir a saúde do paciente e do profissional em atendimento, a continuidade e a qualidade do tratamento e, por fim, a resolubilidade do caso.

Por fim, considerando o impacto da pandemia do coronavírus gerada em toda a Rede de Atenção à Saúde no Brasil, este estudo objetivou levantar na literatura os desafios enfrentados pela APS em relação ao atendimento das demandas específicas de pacientes acometidos pela COVID-19. Dessa forma, pretendese expor a realidade acerca da repercussão da doença nos Centros de Saúde e, consequentemente, na população adscrita a eles.

\section{METODOLOGIA}

Trata-se de uma revisão integrativa da literatura realizada por meio das seguintes etapas: I) identificação do tema de interesse, II) elaboração da pergunta norteadora, III) escolha dos critérios de inclusão e exclusão, IV) determinação da amostra de pesquisa, V) categorização dos estudos, VI) análise da amostra, VII) interpretação e síntese dos resultados obtidos nos estudos.

A elaboração da pergunta norteadora foi embasada nas etapas da metodologia PICo: (P) população; (I) interesse e (Co) contexto, permitindo organizar e sistematizar o tema estudado a partir da formulação de uma pergunta problema, de forma a selecionar criteriosamente os artigos na literatura. Considerou-se os itens: População: pacientes com COVID, Interesse: capacidade de suprir demandas e o Contexto: Atenção Primária. O problema tido como guia deste estudo foi "a Atenção Primária é capaz de suprir a demanda de pacientes com COVID-19?".

Para a estruturação e elaboração do presente artigo, foi utilizada a recomendação Preferred Reporting Items for Systematic Reviews and Meta-Analysis (PRISMA), com o objetivo de aumentar a 
qualidade do artigo, ainda que o mesmo não trate de uma revisão sistemática e não inclua meta-análises em sua composição. Em seguida, foram escolhidos os descritores: Atenção primária, Atenção Básica à saúde, Covid-19 e Brasil; e seus respectivos descritores em inglês. Em cada busca foram utilizados os operadores booleanos "AND" e "OR".

Após a definição, foi realizada a busca de artigos em plataformas online conceituadas como Scientific Electronic Library Online (SciELO), PubMed, Library of Medicine National Institutes of Health (MEDLINE) e Literatura Latino-americana e do Caribe em Ciências da Saúde (LILACS). Primeiro, a seleção de artigos foi realizada com a leitura de resumos e títulos e, posteriormente, a leitura completa dos trabalhos. Foram extraídos dados e contextos característicos dos artigos que fossem relacionados à temática desejada para comparação.

A seleção dos artigos foi realizada no mês de agosto de 2020. Os critérios de inclusão foram artigos originais, de livre acesso, publicados de janeiro a agosto de 2020, nos idiomas português e inglês, que respondessem à pergunta norteadora. Foram excluídos artigos de reflexão, editoriais, revisões de literatura não sistemáticas, cartas, livros, manuais e outros textos que não abordavam especificamente o objeto de estudo, além de artigos duplicados.

Foram encontradas 65 publicações, sendo 27 artigos localizados na base de dados da LILACS, 4 da SciELO, 7 da MEDLINE e 13 do PubMed. Foram excluídas da amostra 12 publicações, pois se tratavam de repetições e 46 artigos que não abordavam o assunto de interesse ou não respondiam à pergunta norteadora do estudo. A amostra final, portanto, foi de 7 artigos (Figura 1). 
Figura 1 - Fluxograma PRISMA dos artigos incluídos na revisão integrativa da literatura.

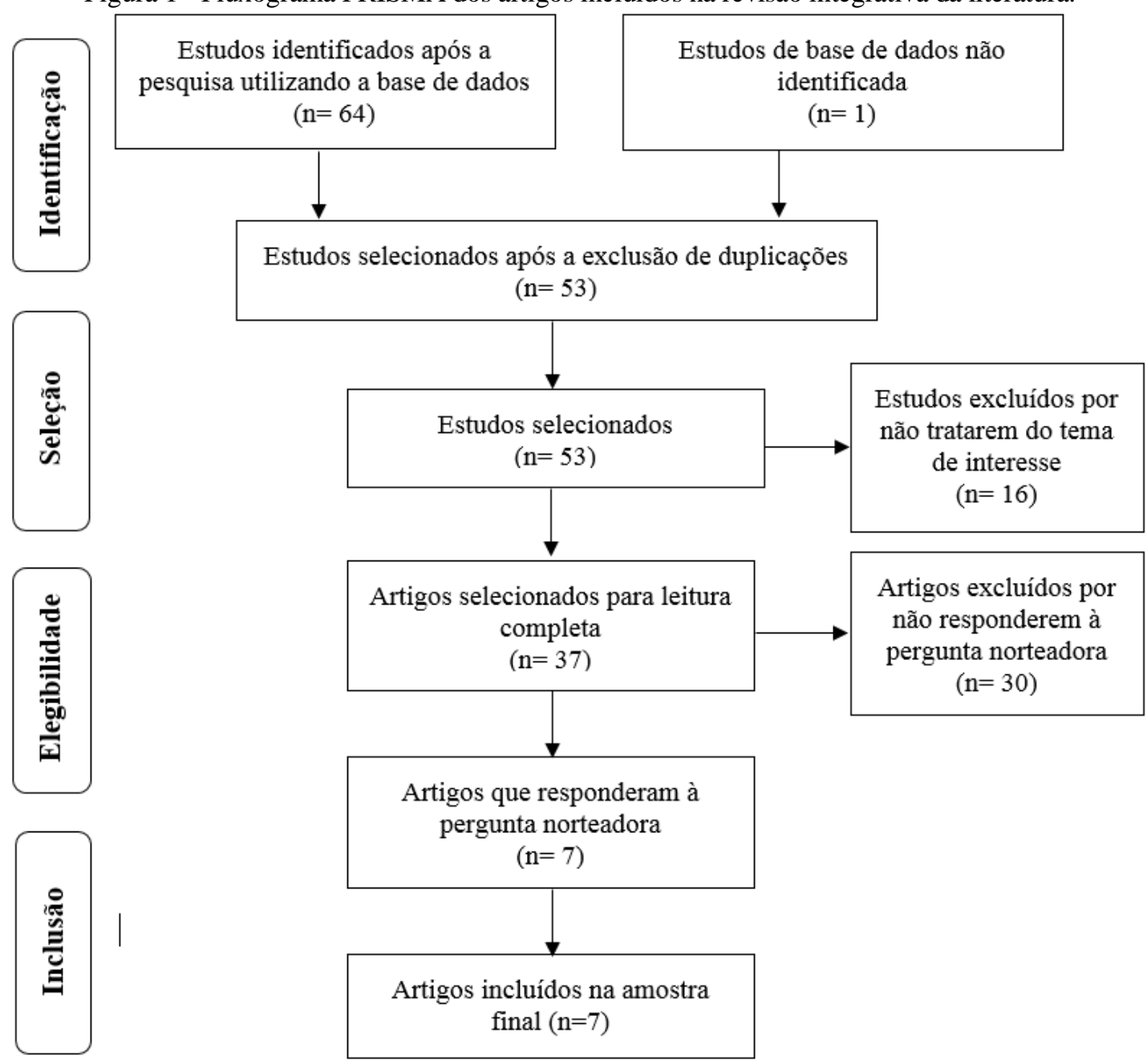

Fonte: Dados da pesquisa, 2020.

Após a obtenção da amostra final dos artigos, criou-se um quadro sinóptico para organização das informações presentes em cada publicação incluída nesta revisão. Considerando o fato de que este estudo não envolveu pesquisa com seres humanos, foi dispensada aprovação pelo Comitê de Ética em Pesquisa (CEP).

\section{RESULTADOS}

Foram incluídos sete artigos publicados na língua inglesa e portuguesa, disponibilizados nas plataformas PubMed, SciELO, LILACS e MEDLINE. O quadro 1 indica a síntese dos estudos que compõem a amostra. 
Quadro 1: Síntese dos artigos selecionados para o estudo, 2020.

\begin{tabular}{|c|c|c|c|}
\hline Título & Autores & Periódico & $\begin{array}{l}\text { Síntese das conclusões/ } \\
\text { recomendações }\end{array}$ \\
\hline $\begin{array}{l}\text { Covid-19 is causing } \\
\text { the collapse of } \\
\text { Brazil's national } \\
\text { health service. }\end{array}$ & $\begin{array}{l}\text { ANDRADE, } \\
\text { Rodrigo. }\end{array}$ & $\begin{array}{l}\text { BMJ; 370: m3032, } \\
\text { jul 2020. }\end{array}$ & $\begin{array}{l}\text { Apesar do acesso à saúde no Brasil ser } \\
\text { fornecido como um serviço público e } \\
\text { universal, a atenção primária } \\
\text { historicamente enfrenta dificuldades } \\
\text { relacionadas a negligência e } \\
\text { subfinanciamento. A chegada da pandemia } \\
\text { do Coronavírus evidenciou ainda mais as } \\
\text { falhas quanto ao fornecimento de } \\
\text { atendimentos e estoque de recursos. }\end{array}$ \\
\hline $\begin{array}{l}\text { What is the role of } \\
\text { Primary Health Care } \\
\text { in the COVID-19 } \\
\text { pandemic? }\end{array}$ & $\begin{array}{l}\text { SARTI, Thiago } \\
\text { D. et al. }\end{array}$ & $\begin{array}{l}\text { Epidemiol. } \\
\text { Saúde vol.29 no.2 } \\
\text { Brasília } 2020 \text { Epub } \\
27 \text { abr } 2020\end{array}$ & $\begin{array}{l}\text { A atenção primária à saúde no Brasil tem } \\
\text { como finalidade oferecer um atendimento } \\
\text { seguro e de qualidade. A desvalorização e } \\
\text { desinvestimento na área da ciência, } \\
\text { tecnologia e saúde levam à precarização do } \\
\text { serviço oferecido, agravando o acesso à } \\
\text { saúde pública pela população diante da } \\
\text { pandemia do Coronavírus. }\end{array}$ \\
\hline $\begin{array}{l}\text { Health equity and } \\
\text { COVID-19: global } \\
\text { perspectives }\end{array}$ & $\begin{array}{l}\text { SHADMI, } \\
\text { Efrat. et al. }\end{array}$ & $\begin{array}{l}\text { Int. J. Equity in } \\
\text { Health. Vol 19, jun- } \\
\text { jul. } 2020 \text {. }\end{array}$ & $\begin{array}{l}\text { A pandemia do Coronavírus representa um } \\
\text { impacto no acesso à saúde pública no } \\
\text { Brasil. Considerando que este serviço } \\
\text { deveria suprir a demanda da grande maioria } \\
\text { dos brasileiros, vê-se que a realidade da } \\
\text { população pobre e vulnerável é de descaso } \\
\text { quanto a qualidade do atendimento } \\
\text { oferecido. }\end{array}$ \\
\hline $\begin{array}{l}\text { The role of primary } \\
\text { care in the Brazilian } \\
\text { healthcare system: } \\
\text { limits } \\
\text { possibilities and } \\
\text { fighting COVID- } 19 .\end{array}$ & $\begin{array}{l}\text { DAUMAS, } \\
\text { Regina P. et al. }\end{array}$ & $\begin{array}{l}\text { Cad. Saúde Pública. } \\
\text { Rio de Janeiro; } \\
\text { 36(6): e00104120, } \\
\text { 26 Jun, } 2020 .\end{array}$ & $\begin{array}{l}\text { Apesar da grande importância do SUS no } \\
\text { enfrentamento da pandemia, sua atuação } \\
\text { enfrenta dificuldades pelo baixo } \\
\text { financiamento, pela necessidade de } \\
\text { implantação de fluxos de atendimento e } \\
\text { reorganização dos centros de saúde, pela } \\
\text { insustentabilidade da APS, pela dificuldade } \\
\text { de implantação da telemedicina e pela falta } \\
\text { de integração na comunicação entre as } \\
\text { redes de atenção. }\end{array}$ \\
\hline $\begin{array}{l}\text { Reorganização da } \\
\text { atenção primária à } \\
\text { saúde para vigilância } \\
\text { universal e contenção } \\
\text { da COVID-19 }\end{array}$ & $\begin{array}{l}\text { TEIXEIRA, } \\
\text { Maria G. et al. }\end{array}$ & $\begin{array}{l}\text { Epidemiol. Serv. } \\
\text { Saúde vol.29 no.4 } \\
\text { Brasília 2020; Epub } \\
03 \text { ago 2020. }\end{array}$ & $\begin{array}{l}\text { A efetividade das medidas de contenção da } \\
\text { pandemia tem sido limitada, } \\
\text { principalmente pelos obstáculos } \\
\text { enfrentados na implementação rigorosa e } \\
\text { ampla de ações que exigem mudanças de } \\
\text { hábitos individuais e coletivos. } \\
\text { Dificuldades quanto à organização das } \\
\text { unidades de atendimento, às falhas na } \\
\text { realização da vigilância epidemiológica e, } \\
\text { novamente, a presença da má gestão } \\
\text { financeira em relação à distribuição de } \\
\text { verba para o sistema de saúde, influenciam } \\
\text { diretamente na efetividade da APS em seu } \\
\text { nicho de atuação. }\end{array}$ \\
\hline
\end{tabular}




\begin{tabular}{|c|c|c|c|}
\hline $\begin{array}{l}\text { The need to } \\
\text { strengthen Primary } \\
\text { Health Care in Brazil } \\
\text { in the context of the } \\
\text { COVID-19 pandemic. }\end{array}$ & $\begin{array}{l}\text { SOUZA, Carlos } \\
\text { DF de et al. }\end{array}$ & $\begin{array}{l}\text { Braz. oral res. São } \\
\text { Paulo; 34: e047, } 11 \\
\text { Mai, 2020. }\end{array}$ & $\begin{array}{l}\text { A APS está se fragilizando nos últimos } \\
\text { anos devido à falta de recursos destinados a } \\
\text { ela. É papel do governo melhorar/aumentar } \\
\text { os recursos necessários para que as APS } \\
\text { consigam combater efetivamente a } \\
\text { pandemia e as demandas diárias da } \\
\text { população, visto sua grande importância } \\
\text { não só no combate à COVID-19, mas } \\
\text { também à diversas outras doenças. }\end{array}$ \\
\hline $\begin{array}{l}\text { Federal actions to } \\
\text { support and } \\
\text { strengthen local } \\
\text { efforts to combat } \\
\text { COVID-19: Primary } \\
\text { Health Care (PHC) in } \\
\text { the driver's seat. }\end{array}$ & $\begin{array}{l}\text { HARZHEIM, } \\
\text { Erno. et al. }\end{array}$ & $\begin{array}{l}\text { Ciênc. saúde } \\
\text { coletiva vol.25 } \\
\text { supl.1 } \\
\text { Rio de Janeiro } 2020 \\
\text { Epub 05 jun } 2020\end{array}$ & $\begin{array}{l}\text { O Ministério da Saúde desenvolveu } \\
\text { estratégias de combate à pandemia. Com } \\
\text { tais recursos é possível aprimorar áreas de } \\
\text { atendimento que vem sendo fundamentais } \\
\text { para o enfrentamento da pandemia, como a } \\
\text { consolidação da telemedicina e a } \\
\text { efetividade da vigilância epidemiológica } \\
\text { que, se mantidas na situação atual, podem } \\
\text { representar um obstáculo na capacidade da } \\
\text { APS de lidar com esse contexto. }\end{array}$ \\
\hline
\end{tabular}

De maneira geral, os estudos discursavam sobre a importância da atenção primária à saúde na linha de frente do atendimento aos pacientes com COVID-19, sem relatar, especificamente como tema central, as dificuldades da APS nesse contexto. No entanto, após a leitura flutuante das informações, foi possível elencar algumas necessidades desse nível de atendimento frente à crise do coronavírus e, com isso, selecionar os trabalhos que guiariam a resposta para o questionamento acerca da capacidade da Atenção Primária à Saúde em relação à pandemia.

Dentre os sete artigos obtidos, três abordavam a questão da telessaúde e suas restrições (DAUMAS et al., 2020; HARZHEIM, 2020; SARTI et al., 2020), seis abordavam o recurso financeiro escasso dos serviços de saúde como dificultadores (ANDRADE et al., 2020; DAUMAS et al., 2020; SARTI et al., 2020; SHADMI et al., 2020; SOUZA et al., 2020; TEIXEIRA et al., 2020), dois incluíam a ineficiência da vigilância epidemiológica nos centros de saúde (HARZHEIM, 2020; TEIXEIRA et at., 2020) e quatro apresentaram a falta de planejamento e organização da APS como fator prejudicial ao cuidado voltado para pacientes com COVID-19 (DAUMAS et al., 2020; SARTI et al., 2020; TEIXEIRA et al., 2020; SOUZA et al., 2020). Assim, a pesquisa fundamentada nos métodos anteriormente expostos sugere que, apesar de o nível primário de acolhimento ser de extrema importância para a contenção do cenário pandêmico, a APS apresenta gargalos originados pela situação em que está sendo posta à prova e tais obstáculos refletem diretamente na qualidade do atendimento oferecido aos pacientes da COVID-19, podendo interferir na sua capacidade de suprir todas as demandas iniciais desse tipo de paciente. 


\section{DISCUSSÃO}

O sistema de saúde público, também conhecido como SUS, foi disponibilizado pelo governo brasileiro e promulgado como um direito aos cidadãos do país em 1998 (BRASIL, c2013b). Tal sistema enfrenta, mesmo depois de anos da sua implantação, problemas devido ao insuficiente investimento financeiro, que reflete no sucateamento desse serviço (SBP, 2020). Ao analisar o impacto dessa falha no combate da COVID-19, fica claro que apesar de se tratar do principal atendimento na linha de frente da pandemia, a atenção primária à saúde não é capaz de suprir todas as demandas de controle da transmissibilidade e do tratamento da infecção pelo vírus. Em comparação com a disponibilidade de leitos no sistema público e privado para o contexto pandêmico, o SUS conta apenas com cerca de um terço dos leitos oferecidos pelas instituições particulares, tal número pode ser mais comprometido caso seja analisado a proporção entre a disponibilidade na região sudeste e a região norte e nordeste do Brasil (SHADMI et al., 2020).

No geral, tem-se a percepção deste sistema de saúde como falho devido a seu caráter histórico de subfinanciamento, não havendo de fato apoio político e esquematização da dinâmica econômica brasileira que consigam suprir a falta de recursos e garantir a seguridade social dos cidadãos que usufruem deste direito (ANDRADE et al., 2020; FUNCIA, 2019; SHADMI et al., 2020). Esta questão enfrentou mais um agravante com a aprovação do teto de gastos para saúde e educação em 2016, diminuindo ainda mais o investimento nos serviços de saúde (ANDRADE et al., 2020; SARTI et al., 2020). A aprovação do congelamento de gastos nesses setores acarretou sérios prejuízos a grande parte da população brasileira, já que além de $72 \%$ da população contam apenas com o acesso público a serviços médicos hospitalares disponibilizados pelo SUS, o financiamento destinado à saúde pública representa apenas 3,4\% do PIB, aproximadamente metade dos recursos disponibilizados em outros países com sistema similar (MARQUES, 2017).

Por ser um país com um grande percentual de cidadãos em situação de pobreza, o Brasil conta com cerca de R \$ 30 milhões de pessoas sem acesso a condições mínimas de saneamento básico e 11,4 milhões que residem em favelas ou outros aglomerados populacionais (BELLO, 2017), o que dificulta o seguimento das normas de higiene, isolamento social e prevenção do coronavírus. Ainda sob o contexto de pandemia, acrescenta-se a questão que engloba a ausência de menção clara de investimento de recursos financeiros ou o fortalecimento do SUS diante desse cenário (SOUZA et al., 2020), visto que mesmo que o Governo tenha liberado cerca de $\mathrm{R} \$ 44,2$ bilhões para a saúde no início da pandemia, apenas 28,3\% desse montante havia sido gasto até o final de julho (ANDRADE et al., 2020). Essa questão levanta questionamentos sobre o porquê, mesmo com tantas evidências de necessidade de recurso, este não está sendo usado. Além disso, ao considerar a prevalência de doenças crônicas que podem ocasionar agravamento da sintomatologia da 
infecção por COVID-19 nessa população, há uma exigência de maiores gastos públicos a nível primário, o que piora a situação de escassez de recursos destinados a esse sistema de saúde (SHADMI et al., 2020).

Como forma de conter a disseminação do vírus, a exitosa estratégia internacional de implantação da telemedicina torna esta uma das melhores ferramentas no enfrentamento da COVID-19, uma vez que protege tanto os funcionários da APS quanto os pacientes de uma possível contaminação devido à quebra do isolamento social (HARZHEIM, 2020; CABRAL, 2020). Contudo, o acesso e a disponibilidade da tecnologia necessária e a capacitação dos profissionais para o uso da mesma não é a realidade do serviço público de saúde do Brasil, limitando essa forma de atendimento pela atenção básica por falta de recursos nas unidades de saúde (CABRAL, 2020).

Ao apresentar a baixa disponibilidade de recursos financeiros destinados às APS, pelo Ministério da Saúde, para completo funcionamento do teleatendimento à população, destaca-se, ainda mais, como esse modelo é falho e prejudica a assistência aos pacientes suspeitos e/ou acometidos pela COVID-19 (SARTI et al., 2020). A escassez financeira provoca a falta de recursos necessários para a construção de tendas de suporte externo para atendimento à população, bem como a ausência de internet de boa qualidade para unidades de saúde e para os pacientes em geral, além da indisponibilidade de celulares e outros aparelhos necessários para a completa assistência à população (DAUMAS et al., 2020; SARTI et al., 2020). Todos os fatores citados anteriormente são decisivos para que ocorra a ampla e correta implementação da telemedicina na APS, como forma de melhorar a triagem de casos e tornar eficaz o contato entre a unidade básica e os centros de atenção de maior complexidade em casos de encaminhamento de pacientes (DAUMAS et al., 2020).

Ainda sobre a problemática da ineficiência da telessaúde, é necessário que esse sistema traga confiança aos profissionais de saúde que o utilizam, uma vez que a incerteza sobre a qualidade dessa forma de atendimento, a ausência de computadores disponíveis nas unidades, a inexistência de um prontuário integrado disponível em formato eletrônico e o impedimento para emissão de receitas e realização de solicitações de exames para os pacientes provocam dúvidas sobre sua aplicação eficaz pelos profissionais da área (SARTI et al., 2020). Dessa forma, é importante ressaltar que esse problema se relaciona tanto à falta de recursos financeiros disponíveis para implementar essa modalidade de atendimento quanto à necessidade de melhorar o sistema como um todo, preparar e treinar os profissionais para o uso, intensificar a organização da equipe destinada a esse serviço e promover o planejamento necessário para atender a população geral.

A má composição e o irregular gerenciamento da APS também contribuem diretamente para que haja prejuízos no atendimento amplo aos pacientes com COVID-19. Por mais que o MS tenha criado fluxogramas de assistência específicos para o período de pandemia, é reconhecido que há uma limitação na aplicação e no resultado obtido pela implementação desses métodos, visto que os centros de saúde carregam 
grandes deficiências em um cenário de normalidade e não seria diferente durante o enfrentamento dessa situação emergencial (DAUMAS et al., 2020; SOUZA et al., 2020). Esse quadro se intensifica ao identificar que existe falha na administração entre os níveis assistenciais, ausência de fluxos entre toda a rede de atenção, escassez da aplicação de estratégias de cuidado aos pacientes, falta de adaptação aos protocolos de acordo com as características epidemiológicas da doença e de definição desses protocolos para poder acolher os pacientes suspeitos e acometidos pela infecção (DAUMAS et al., 2020; SARTI et al., 2020; SOUZA et al., 2020). Dessa maneira, torna-se evidente como é necessário que haja planejamento e organização na APS para que se possa realizar o correto manejo à população diante esse momento atípico vivido em todo o mundo, além da importância dessa modalidade de cuidado na contribuição no controle da doença e diminuição da quantidade de óbitos e casos confirmados no Brasil.

Além dos obstáculos supracitados, há ainda falhas no que tange a vigilância epidemiológica do vírus no Brasil. Como plano inicial, a APS deveria, além de fornecer o atendimento aos pacientes infectados, preparar a pesquisa sorológica para COVID-19 em domicílio como forma de evitar a exposição de contaminados assintomáticos ou com quadro leve e realizar também a pesquisa telefônica para identificar possíveis novos casos gripais. Paralelamente a essas medidas, deveria haver o cadastramento de 210 milhões de brasileiros no cadastro nacional do SUS para melhor monitoração da população (TEIXEIRA et al., 2020). Porém, sem o emprego de tecnologias atualizadas e de acesso comum entre as unidades da APS, como é o percebido na prática do serviço de saúde público brasileiro, a realização do perfil epidemiológico do país associando-se a base de dados de diferentes regiões fica comprometido, sendo divulgado apenas resultados que contemplam casos confirmados laboratorialmente. Com isso, os dados referentes a essa pesquisa são capazes de mostrar a extensão e magnitude da atual pandemia, o que compromete a logística de prevenção, acompanhamento e tratamento da população (SOUZA et al., 2020).

No atual cenário, a Secretaria de Vigilância em Saúde é responsável por coordenar a investigação de qualquer doença transmissível de impacto nacional, sendo a associação de seu trabalho com a organização da metodologia empregada pela APS fundamental para efetividade do trabalho deste serviço de saúde. Assim, ao considerar a ocorrência de erros na testagem para rastreio e diagnóstico dos brasileiros, não há o conhecimento preciso do fluxo de contaminação pelo Coronavírus (SOUZA et al., 2020).

Em resumo, propõe-se que, assim como ocorre no SUS em situações alheias ao contexto de pandemia, a falta de investimento financeiro para a saúde desencadeia problemas na APS que são capazes de interferir na cadeia de atendimento em níveis superiores ao primário, tornando as consequências dessa negligência cada vez mais altas (ANDRADE et al., 2020). É possível inferir que dificuldades anteriormente citadas como a implementação da telemedicina, a falha na vigilância epidemiológica e problemas de planejamento organizacional das unidades de atendimento, além da própria escassez de recursos, envolvem, 
substancialmente, o baixo e até inexistente (comparado ao necessário) investimento de capital no sistema público de saúde.

Ao expor as dificuldades enfrentadas pela Atenção Primária frente a pandemia provocada pelo coronavírus, apresentando os pontos já discutidos anteriormente como fatores ligados diretamente à ineficiência da abordagem aos pacientes com COVID-19, é possível compreender quais pontos devem ser melhorados nesse momento atípico para que o atendimento seja considerado justo e apropriado. Desse modo, a garantia de atendimento integral deve ser regida pelos princípios que buscam: melhorar a organização e planejamento das equipes dos centros de saúde, aumentar o suporte financeiro, melhorar a capacitação dos funcionários, assegurar a criação de espaços de acolhimento de pacientes, garantir a quantidade ideal de profissionais nas equipes e disponibilizar equipamentos de proteção individual para todos os trabalhadores e pacientes sintomáticos, entre diversas outras questões que extrapolam a discussão apresentada por este trabalho (SARTI et al., 2020). Portanto, acredita-se que apontar os obstáculos existentes ao longo de todo o processo de cuidado possibilita a busca por melhorias em todos os segmentos da APS, permitindo o estabelecimento de uma política de atenção à saúde concreta, segura e, principalmente, funcional.

\section{CONSIDERAÇÕES FINAIS}

Esse artigo levantou na literatura os desafios enfrentados pela APS em relação ao atendimento das demandas específicas de pacientes acometidos pela COVID-19. Destaca-se que as dificuldades enfrentadas pela APS durante a pandemia são reflexos da gestão da saúde pública em um cenário anterior à tal crise sanitária. Entende-se que o fato de o sistema de saúde brasileiro conviver com irregularidades políticoadministrativas em seu estado normal de funcionamento faz com que, ao se instalar uma crise, ocorra um grau de colapso superior ao esperado, principalmente se comparado ao nível de desordem cabível em um contexto prévio no qual o gerenciamento do sistema é bem conduzido em sua esfera financeira e burocrática. Dessa forma, a má condução do sistema sanitário em sua conjuntura normal, sem interferência de uma pandemia, resulta em consequências exponencialmente maiores quando esta se instala.

Por fim, é possível concluir que a APS brasileira, se não tivesse corrompido os princípios definidos em Alma-Ata (1978), seria capaz de lidar com a crise gerada pelo SARS-CoV-2 sem somar às dificuldades inerentes à pandemia os problemas que deveriam ter sido sanados assim que surgiram. Portanto, tem-se uma resposta confirmatória ao questionar se a capacidade da atenção primária no acolhimento das necessidades do paciente com a COVID-19 se mostra comprometida, principalmente quando se leva em consideração a realidade brasileira em relação ao seu sistema de saúde no contexto pré pandêmico, no qual já eram observadas complicações significativas que repercutem de forma ainda mais incisiva quando se instala uma situação intrinsecamente prejudicial como a pandemia. 


\section{REFERÊNCIAS}

ANDRADE, Rodrigo O de. Covid-19 is causing the collapse of Brazil's national health service. BMJ, São Paulo, v. 370, n. 3032, 2020. Disponível em: 〈https://doi.org/10.1136/bmj.m3032〉. Acesso em: 18 ago. 2020.

BELLO, Luiz. Dia Nacional da Habitação: Brasil tem 11,4 milhões de pessoas vivendo em favelas. Agência IBGE Notícias. 21 ago. 2017. Disponível em: <https://agenciadenoticias.ibge.gov.br/agencianoticias/2012-agencia-de-noticias/noticias/15700-dados-do-censo-2010-mostram-11-4-milhoes-de-

pessoas-vivendo-em-favelas>. Acesso em: 18 ago. 2020.

BRASIL. Ministério da Saúde. Atenção Primária. c2013a. Disponível em: <https://antigo.saude.gov.br/atencao-primaria>. Acesso em: 18 ago. 2020.

BRASIL. Ministério da Saúde. Coronavírus Brasil. Painel de casos de doença pelo coronavírus 2019 (COVID-19) no Brasil pelo Ministério da Saúde. 2020a. Disponível em: 〈https://covid.saude.gov.br/>. Acesso em: 18 ago. 2020.

BRASIL. Ministério da Saúde. Declaração de Alma-Ata. In: Conferência Internacional sobre Cuidados Primários de Saúde. 1978, Alma Ata.

BRASIL. Ministério da Saúde. Orientações para Manejo de Pacientes com Covid-19. Brasília: Ministério da Saúde, 2020b. 49 p.

BRASIL. Ministério da Saúde. Sistema Único de Saúde. c2013b. Disponível em: <https://antigo.saude.gov.br/sistema-unico-de-saude>. Acesso em: 25 ago. 2020.

CABRAL, Elizabeth R. M., et al. Contributions and challenges of the Primary Health Care across the pandemic COVID-19. InterAmerican Journal of Medicine and Health, Seção COVID-19 em Debate, v. 3, p. 1 - 12, 11 abr. 2020. Disponível em: 〈https://iajmh.com/iajmh/article/view/87>. Acesso em: 18 ago. 2020.

DAUMAS, Regina P. et al. The role of primary care in the Brazilian healthcare system: limits and possibilities for fighting COVID-19. Cadernos de Saúde Pública, Rio de Janeiro, v. 36, n. 6, e00104120, 2020. Disponível em: <http://www.scielo.br/scielo.php?script=sci_arttext\&pid=S0102311X2020000600503\&lng=en\&nrm=iso>. Acesso em: 18 ago. 2020. Epub June 26, 2020. https://doi.org/10.1590/0102-311x00104120.

FUNCIA, Francisco R. Subfinanciamento e orçamento federal do SUS: referências preliminares para a alocação adicional de recursos. Ciência e Saúde coletiva, Rio de Janeiro, v. 24, n. 12, p. 4405-4415, 2019. Disponível em:

<http://www.scielo.br/scielo.php?script=sci_arttext\&pid=S1413-81232019001204405\&lng=en>. Acesso em: 25 ago. 2020.

GIOVANELLA, Lígia. Atenção básica ou atenção primária à saúde?. Cadernos de Saúde Pública, v. 34, p. e00029818, 2018. Disponível em: <http://cadernos.ensp.fiocruz.br/static/arquivo/1678-4464-csp-34-08e00029818.pdf>. Acesso em: 18 ago. 2020.

HARZHEIM, Erno. et al. Ações federais para apoio e fortalecimento local no combate ao COVID-19: a Atenção Primária à Saúde (APS) no assento do condutor. Ciência \& saúde coletiva, Rio de Janeiro, v. 25, 
supl. $\quad 1, \quad$ p. 2493-2497, 2020. Disponível em: $<$ http://www.scielo.br/scielo.php?script=sci_arttext\&pid=S1413$81232020006702493 \& \operatorname{lng}=$ en\&nrm=iso >. Acesso em: 18 ago. 2020.

JOHNS HOPKINS UNIVERSITY. Coronavirus COVID-19 Global Cases by Johns Hopkins CSSE. 2020. Disponível em: <https://coronavirus.jhu.edu/map.html>. Acesso em: 18 ago. 2020.

MARQUES, Rosa M. Notas Exploratórias Sobre as Razões do Subfinanciamento Estrutural do SUS. Planejamento e Políticas Públicas, n. 49. Jul/dez 2017. Disponível em: <https://www.ipea.gov.br/ppp/index.php/PPP/article/view/950/442>. Acesso em: 25 ago. 2020.

MEDINA, Maria Guadalupe et al. Atenção primária à saúde em tempos de COVID-19: o que fazer?. Cadernos de Saúde Pública, v. 36, p. e00149720, 2020. Disponível em: <http://cadernos.ensp.fiocruz.br/static/arquivo/CSP_1497_20_Atencao_pt.pdf>. Acesso em: 18 ago. 2020. OLIVEIRA, Maria A.C.; PEREIRA, Iara C. Atributos essenciais da Atenção Primária e a Estratégia Saúde da Família. Revista Brasileira de Enfermagem, Brasília, v. 66, n. spe, p. 158-164, 2013. Disponível em: $<$ http://www.scielo.br/scielo.php?script=sci_arttext\&pid=S0034-

71672013000700020\&lng=en\&nrm=iso>. Acesso em: 18 ago. 2020. http://dx.doi.org/10.1590/S003471672013000700020 .

PORTELA, Margareth C. et al. Matriz Linha de Cuidado Covid-19 na Rede de Atenção à Saúde. Observatório Covid-19. FIOCRUZ. 2020. 15 p.

ROTHAN, Hussin A. et al. The epidemiology and pathogenesis of coronavirus disease (COVID-19) outbreak. Journal of autoimmunity, p. 102433, 2020. Disponível em: <https://www.sciencedirect.com/science/article/pii/S0896841120300469>. Acesso em: 18 ago. 2020.

SARTI, Thiago D. et al. Qual o papel da Atenção Primária à Saúde diante da pandemia provocada pela COVID-19?. Epidemiologia e Serviços de Saúde, Brasília, v.

29, n. 2, e2020166, 2020. Disponível em: <http://www.scielo.br/scielo.php?script=sci_arttext\&pid=S223796222020000200903\&lng=en\&nrm=iso>. Acesso em: 18 ago. 2020.

SBP. Sociedade Brasileira de Pediatria. Falta de plano de carreira e subfinanciamento do SUS prejudica a saúde brasileira. Disponível em: <https://www.sbp.com.br/sbp-servicos/falta-de-plano-de-carreira-esubfinanciamento-do-sus-prejudica-a-saude-brasileira/>. Acesso em: 18 de ago. 2020.

SHADMI, Efrat. et al. Health equity and COVID-19: global perspectives. International Journal for Equity Health, [s.1], v. 19, n. 104. p. 2-14, 2020. Disponível em: 〈https://doi.org/10.1186/s12939-020-01218-z〉. Acesso em: 25 ago. 2020.

SOUZA, Carlos DF de et al. The need to strengthen Primary Health Care in Brazil in the context of the COVID-19 pandemic. Brazilian oral research, São Paulo, v. 34, e. 047, 2020. Disponível em: <https://www.scielo.br/scielo.php?pid=S1806-83242020000100801\&script=sci_abstract $>$. Acesso em: 25 ago. 2020.

STARFIELD, Bárbara. Atenção primária: equilíbrio entre necessidade de saúde, serviços e tecnologia. Brasília: UNESCO: Ministério da Saúde. 726p. 2004.

TEIXEIRA, Maria G. et al. Reorganização da atenção primária à saúde para vigilância universal e contenção da COVID-19. Epidemiologia e Serviços de Saúde, Brasília , v. 29, n. 4, e 2020494, 2020. 
Disponível

em:

<https://www.scielo.br/scielo.php?pid=S2237-

96222020000400900\&script=sci_arttext\&tlng=pt>. Acesso em: 18 ago. 2020.

WORLD HEALTH ORGANIZATION. Oxygen sources and distribution for COVID-19 treatment centres: interim guidance. Geneva: World Health Organization, 2020. 